\title{
Pharmacokinetics and Pharmacodynamics of Vancomycin in Severe COVID-19 Patients: a Preliminary Study in a Chinese Tertiary Hospital
}

Lin Yin

Shanghai Public Health Clinical Center, Fudan University

Tangkai Qi

Shanghai Public Health Clinical Center, Fudan University

Yuancheng Chen

Huashan Hospital, Fudan University

Mingquan Guo

Shanghai Public Health Clinical Center, Fudan University

Huichun Shi

Shanghai Public Health Clinical Center, Fudan University

Yaxin Fan

Huashan Hospital, Fudan University

Yun Ling

Shanghai Public Health Clinical Center, Fudan University

Yonghong Tao

Shanghai Public Health Clinical Center, Fudan University

Yili Li

Shanghai Public Health Clinical Center, Fudan University

Lin Wang

Shanghai Public Health Clinical Center, Fudan University

Menglu Gao

Shanghai Public Health Clinical Center, Fudan University

Shuibao Xu

Shanghai Public Health Clinical Center, Fudan University

Xianmin Meng

Shanghai Public Health Clinical Center, Fudan University

Jin Ke

Shanghai Public Health Clinical Center, Fudan University

Junjun Ping

Shanghai Public Health Clinical Center, Fudan University

Yaru Xing

Shanghai Public Health Clinical Center, Fudan University

Wenhong Zhang

Huashan Hospital, Fudan University

Zhaoqin Zhu ( $\nabla$ zhaoqinzhu@163.com ) 
Shanghai Public Health Clinical Center, Fudan University

Jing Zhang ( $\sim$ zhangj_fudan@163.com )

Huashan Hospital, Fudan University

Hongzhou Lu ( $\nabla$ luhongzhou@fudan.edu.cn )

Shanghai Public Health Clinical Center, Fudan University

Lijun Zhang

Shanghai Public Health Clinical Center, Fudan University

\section{Research Article}

Keywords: vancomycin, coronavirus disease 2019, therapeutic drug monitoring, pharmacokinetics, pharmacodynamics

Posted Date: June 24th, 2020

DOI: https://doi.org/10.21203/rs.3.rs-37635/v1

License: (c) (i) This work is licensed under a Creative Commons Attribution 4.0 International License. Read Full License 


\section{Abstract}

Vancomycin plays an important role in the treatment of concurrent infections in severe coronavirus disease 2019 (COVID-19) patients. However, few is known about its pharmacokinetics (PK) in these patients. Here we performed therapeutic drug monitoring (TDM) of intravenous vancomycin with or without nasal administration in these patients. Drug dosage was adjusted depending on vancomycin concentration. A population PK model was developed using NONMEM software. Therapeutic effects, and vancomycin-related adverse events were monitored. A total of 63 samples from 8 patients were analyzed by ultra-performance liquid chromatography-tandem mass spectrometry. The mean trough and peak concentration were 13.79 $\pm 6.61(4.63-34.2) \mathrm{mg} / \mathrm{L}(\mathrm{n}=36)$ and 30.97 $\pm 9.71(17-49.9) \mathrm{mg} / \mathrm{L}$ $(n=27)$, respectively. $25.4 \%$ of serum vancomycin concentration was beyond optimal range. Dose adjustments were made for 3 patients. The PK of vancomycin was consistent with two-compartment model, with the clearance and distribution volume in the central compartment of $4.3 \mathrm{~L} / \mathrm{h}$ and $2.0 \mathrm{~L}$, respectively. The $\mathrm{AUC}_{0-24} / \mathrm{MIC}$ of vancomycin was $848 \pm 566 \mathrm{~h}$. Target infection was clinically cured in all patients, and no vancomycin-associated nephrotoxicity was detected during the TDM process. In conclusion, the PK studies of vancomycin in COVID-19 patients are needed to optimize drug dosage. Based on our PK model, the clearance of vancomycin was $4.3 \mathrm{~L} / \mathrm{h}$.

\section{Introduction}

Since December 2019, a novel coronavirus disease (COVID-19) has been spreading rapidly all over the world, with nearly 3 million confirmed cases and 204 thousand deaths, till April 29, 2020 (https://www.who.int/). Secondary bacterial infections were observed in $31 \%$ of patients who required invasive mechanical ventilation and in $50 \%$ of non-survivors. ${ }^{1}$ Gram-positive bacteria including methicillin-resistant Staphylococcus aureus (MRSA), methicillinresistant coagulase-negative Staphylococci (MRCNS) and Enterococcispecies are common nosocomial pathogens, which mainly cause ventilator-associated pneumonia (VAP). ${ }^{2}$ Vancomycin $15 \mathrm{mg} / \mathrm{kg}$ IV per $8-12 \mathrm{~h}$ with or without a loading dose was recommended for treating such infections. ${ }^{3}$ However, due to the narrow treatment window and personal difference, sub-optimal vancomycin concentrations were prevalent, leading to insufficient antibacterial potency or increased risk of acute kidney injury. ${ }^{4-7}$ Therefore, it is necessary to perform therapeutic drug monitoring (TDM) of vancomycin, so as to ensure its clinical effect while minimizing the occurrence of adverse reactions. ${ }^{8}$ For severe MRSA infection, the guidelines recommended a ratio of 24- hour area under the concentration time curve and minimum inhibitory concentration $\left(\mathrm{AUC}_{0-24} / \mathrm{MIC}\right)$ of 400 to 600 in both adult and pediatric patients to maximize the clinical efficacy and minimize acute kidney injury (AKI) risk. ${ }^{9}$ Because AUCs are not routinely available in clinical practice, plasma or serum concentration is also used as substitute. ${ }^{10-13}$ The guidelines of the Chinese Pharmacological Society recommended a serum trough level of $10-15 \mathrm{mg} / \mathrm{L}$ in adult patients and $10-20 \mathrm{mg} / \mathrm{L}$ for serious MRSA infections. ${ }^{14}$ Furthermore, the peak concentration was expected to be less than $40 \mathrm{mg} / \mathrm{L} .{ }^{15}$

Since the mass hospitalization of patients with COVID-19, including a high proportion relying on mechanical ventilation, it might increase vancomycin usage for treating hospital-acquired infections, especially ventilatorassociated pneumonia (VAP). However, there was little knowledge about the pharmacokinetics of vancomycin in these patients. The decision of drug dosage relied on clinical experiences. Therefore, in this study, we performed TDM of vancomycin by ultra performance liquid chromatography/tandem mass spectrometry (UPLC-MS/MS) in COVID-19 patients. Doses were optimized according to drug concentration.

\section{Results}

\section{Baseline Characteristic and Outcome of COVID-19 Patients}


Among 368 patients hospitalized from Feb 11 to Mar 23, eight $(2.17 \%)$ received intravenous vancomycin treatment. TDM was conducted for all eight patients based on the clinical requirement (Table 1 and Table S1). The median age was 64.5 (57-81) years, including six males and two females (Table 1 and Table S1). Seven (87.5\%) patients had a clear etiology, including four cases with Enterococcus faecium pneumonia, and three with Staphylococcus haemolyticus bacteremia. The rest one received empirical vancomycin treatment for pneumonia. At baseline, each of them was on invasive mechanical ventilation. During the therapeutic process, 5 (62.5\%) were on Extracorporeal Membrane Oxygenation (ECMO), and 4 (50\%) were on hemodyalisis. The baseline creatinine concentration and eGFR were 91.04 (31.67-188.67) $\mu \mathrm{mol} / \mathrm{L}$ and 92.33 (31.7-193.99) mL/min, respectively. Seven out of 8 have basic diseases. The initial vancomycin dosage was $1000 \mathrm{mg}$ every 12 hours (1000 mg Q12 h) in six patients, 500mg per 8 hours (500 mg Q8 h) in one and 1000 mg every $24 \mathrm{~h}(1000 \mathrm{mg} \mathrm{Qd})$ in the last patient. Five of them were also administered vancomycin through nasogastric tube feeding. The median treatment duration (including nasal administration) was 18.6 (5-39) days. All 7 culture confirmed infection turned negative after vancomycin treatment. Among patients who did not receive hemodialysis at baseline, 50 \% (2/4) experienced AKI, including 1 initiated hemodialysis 4 days after vancomycin treatment.

\section{Vancomycin Concentration Determination by UPLC-MS/MS}

Vancomycin concentrations were detected by UPLC-MS/MS (supplement results). The ion channels of vancomycin and demethylvacomycin (IS) were m/z 725.5/144.2, and 718.4/144.2, respectively. As shown in Supplement Figure 1 (Figure S1), vancomycin and IS were eluted at about $1.7 \mathrm{~min}$. The endogenous substances in the blank serum did not interfere with vancomycin and IS (Figure S1A and S1B). The compounds eluted from healthy donors (Figure S1C and S1D) were similar to those from COVID-19 patient's samples (Figure S1E and S1F). The calibration curve range was $1-100 \mathrm{mg} / \mathrm{L}$, and met the clinical requirement.

\section{TDM of Vancomycin in COVID-19 Patients}

A total of 63 time spots were monitored, including 36 troughs and 27 peaks (Table S1). Out of the 36 trough samples, nine had concentrations less than $10 \mathrm{mg} / \mathrm{L}$ and 5 have concentrations greater than $20 \mathrm{mg} / \mathrm{L}$. Of the 27 peak samples, seven had concentrations more than $40 \mathrm{mg} / \mathrm{L}$, and 4 less than $20 \mathrm{mg} / \mathrm{L}$. The mean trough concentration

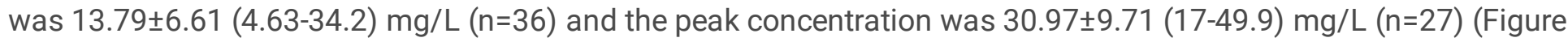
$1 \mathrm{~A})$. For patients with available samples on peak or trough, $28.6 \%(2 / 7))$ patients had at least one trough concentration less than $10 \mathrm{mg} / \mathrm{L}$, and $80.0 \%$ (4/5) of the patients had at least one peak concentration greater than $40 \mathrm{mg} / \mathrm{L}$. Of which, patient No.1 and 2 patients were continuously monitored for 16 days, and thus, more samples were collected from them than from the others, who had one to four samples (Table S1). For No.1, five samples showed trough concentrations beyond the normal range $(10-20 \mathrm{mg} / \mathrm{L})$ and two samples showed higher peak concentration (>40 mg/L) (Figure 1B). For patient No.2, 10 samples (50\%) were out of normal range, including 7 at trough and 3 at peak (Figure 1C).

Furthermore, we examined the data from the first test of each patient, and found that $66.7 \%$ (4/6) of peak concentrations were higher than the upper limit of $40 \mathrm{mg} / \mathrm{L}$ with a mean of 37.19 (17-49.9) mg/L. Furthermore, $55.6 \%$ (5/9) of the trough concentrations were also beyond the recommended range $(10-20 \mathrm{mg} / \mathrm{L})$ with a mean of 15.59 (4.63-26.6) mg/L (Figure 1D).

\section{Dose Adjustment Dependent on Drug Concentration}

Four out of eight (50\%) patients had normal concentrations at the first detection; one (patient No. 5) had a little higher peak concentration $(41.3 \mathrm{mg} / \mathrm{L}$ at peak (Table S1). Dose adjustment of intravenous vancomycin was made 
for the other three (37.5\%) patients (No.1, 2 and 4) according to their serum drug concentrations. After dose adjustments, the peak concentrations $(27.37(17.8-41.7) \mathrm{mg} / \mathrm{L})$ were basically returned to normal range. Significant difference $(P<0.05)$ was detected in peak concentrations before and after dose adjustment (Figure 1E).

The curve of concentration for vancomycin, GFR, and creatinine from three patients with dose adjustments was shown in Figure 2هFigure 2A for Patient No.1, 2B for Patient No.2, 2C for Patient No.4区. Patient No.1 (Figure 2A) was initiated with intravenous vancomycin at $1000 \mathrm{mg}$ per $12 \mathrm{~h}$ to treat Staphylococcus haemolyticus. On day 5 , at first detection, $\mathrm{C}_{\text {trough }}$ was $6.8 \mathrm{mg} / \mathrm{L}$ lower than $10 \mathrm{mg} / \mathrm{L}$. The intravenous dose was then increased to $1000 \mathrm{mg} \mathrm{Q8} \mathrm{h.} \mathrm{He}$ was also given nasal vancomycin at $250 \mathrm{mg} \mathrm{Q12} \mathrm{h} \mathrm{from} \mathrm{day} 7 . \mathrm{C}_{\text {peak }}$ was 47.7 and $41.9 \mathrm{mg} / \mathrm{L}$ on day 7 and day 8 , respectively. Intravenous dose was decreased to $1000 \mathrm{mg} \mathrm{Q12} \mathrm{h} \mathrm{on} \mathrm{day} \mathrm{8,} \mathrm{and} \mathrm{C}_{\text {trough }}$ was $4.63 \mathrm{mg} / \mathrm{L}$ and $6.7 \mathrm{mg} / \mathrm{L}$ on day 11 and day 13, respectively. Intravenous dose was further adjusted to $500 \mathrm{mg}$ Q6 h on day 13 . Since then, optimal drug concentration was detected with $90 \%$ (9/10) of samples on trough spots and $100 \%$ (9/9) on peak spots. However, he met the criteria of grade $1 \mathrm{AKI}$ on day 28 and then stopped intravenous vancomycin.

Patient No. 2 (Figure 2B) was initiated with intravenous vancomycin at $1000 \mathrm{mg} \mathrm{Q12} \mathrm{h}$ to treat Enterococcus faecalis bacteremia. He present with renal dysfunction and was on hemodialysis since baseline. At first detection on day3, $\mathrm{C}_{\text {peak }}$ was $33 \mathrm{mg} / \mathrm{L}$ and intravenous dose was adjusted to $1000 \mathrm{mg}$ Q8h according to improved eGFR. However, $\mathrm{C}_{\text {peak }}$ rised to $46.6 \mathrm{mg} / \mathrm{L}$ on day 4 , then on the same day intravenous vancomycin was stopped. He also received nasal vancomycin from day 5 to day 39 . From day 5 to day $12, C_{\text {peak }}$ and $C_{\text {trough }}$ gradually returned to normal. He was given $1000 \mathrm{mg}$ Q24h of vancomycin intravenously on day 10 and stopped on day 12 , when both $\mathrm{C}_{\text {peak }}$ and $\mathrm{C}_{\text {trough }}$ exceeded the expected range. From day 15 to day $33, C_{\text {trough }}$ was $5.9-13.1 \mathrm{mg} / \mathrm{L}$ and $\mathrm{C}_{\text {peak }}$ was $17.8-30.9 \mathrm{mg} / \mathrm{L}$, although the patient was on nasal vancomycin alone.

Patient No.4 (Figure 2C) was initiated with intravenous vancomycin at 500mg Q8h to treat Enterococcus faecium pneumonia. On day 4 and 5, $\mathrm{C}_{\text {trough }}$ was $26.6 \mathrm{mg} / \mathrm{L}$ and $25.5 \mathrm{mg} / \mathrm{L}$, respectively. After that, vancomycin administration was paused till day 10 , when the patient was given intravenous vancomycin at 500mg Q8h and nasal vancomycin at $250 \mathrm{mg}$ Q6h. On day $12, \mathrm{C}_{\text {trough }}$ and $\mathrm{C}_{\text {peak }}$ were $19.5 \mathrm{mg} / \mathrm{L}$ and $41.7 \mathrm{mg} / \mathrm{L}$, respectively. Intravenous vancomycin was stopped on day 13 , after the blood culture results were negative.

\section{Population PK and pharmacokinetic / pharmacodynamic (PK/PD) analysis}

The PK parameters of vancomycin were shown in Table 2. CL and Q were $4.3 \mathrm{~L} / \mathrm{h}$ and $4.1 \mathrm{~L} / \mathrm{h}$, and $\mathrm{V}_{1}$ and $\mathrm{V}_{2}$ were 2.0 $\mathrm{L}$ and $56.7 \mathrm{~L}$ respectively. Half-life for distribution phase and elimination phase was $10 \mathrm{~min}$ and $19 \mathrm{~h}$, respectively. Hemodialysis and serum creatinine level were covariates on the $\mathrm{CL}$. Both of them were consistent with the power model. The CL in patients with hemodialysis decreased by $58 \%$ compared to those in patient without hemodialysis. IIV of CL was removed because it was close to zero after adding hemodialysis and serum creatinine level as the covariates. ECMO did not have significant effect on vancomycin PK parameters. As shown in Figure 3A, individual predictions were close to observations. The correlation coefficient reached 0.81 . Most of conditional weighted residuals distributed evenly across zero horizontal line (Figure 3B), indicating that the model estimates were reliable and stable.

The $\mathrm{AUC}_{0-24}$ of vancomycin was shown in Table 1 . The mean \pm SD were $622 \pm 218 \mathrm{~h} \cdot \mathrm{mg} / \mathrm{L}$, and coefficient of variation was $35 \%$. If the vancomycin dose was higher, or the drug was given more frequent, the $\mathrm{AUC}_{0-24}$ would increase. For example, in patient No.1, $\mathrm{AUC}_{0-24}$ changed from 871 to $740 \mathrm{~h} \cdot \mathrm{mg} / \mathrm{L}$ when the dosage changed from $1 \mathrm{~g}$ 


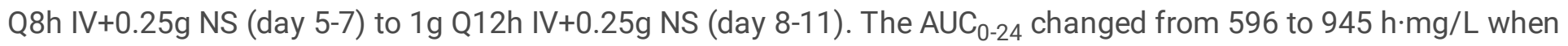
the dosage changed from 1g Q12h IV (day0-2) to 1g Q8h IV (day3) in patient No. 2.

$\mathrm{AUC}_{0-24} / \mathrm{MIC}$ of vancomycin was shown in Table 1 . The mean $\pm \mathrm{SD}$ was $848 \pm 566 \mathrm{~h} \cdot \mathrm{mg} / \mathrm{L}$, and coefficiency of variation was $67 \%$. The maximum and minimum of $\mathrm{AUC}_{0-24} / \mathrm{MIC}$ were 1738 and $244 \mathrm{~h} \cdot \mathrm{mg} / \mathrm{L}$, respectively. Although $\mathrm{AUC}_{0-24} / \mathrm{MIC}$ for 3 patients was less than 400 , the microbiological effects were all successful. There was no correlation between $\mathrm{AUC}_{0-24} / \mathrm{MIC}$ and microbiological effect $\left(\mathrm{R}^{2}=0.01\right) . \mathrm{AUC}_{0-24}$ had a positive correlation with the grade of $A K I$. $A U C_{0-24}=675 \mathrm{~h} \cdot \mathrm{mg} / \mathrm{L}$ was the best critical value for differentiating $A K I$ occurrence. When $A C_{0-24} \geq$ $675 \mathrm{~h} \cdot \mathrm{mg} / \mathrm{L}, 2$ of 3 patients (67\%) had AKI. Meanwhile, When $\mathrm{AUC}_{0-24}<675 \mathrm{~h} \cdot \mathrm{mg} / \mathrm{L}$, only 1 of 5 patients (20\%) had $\operatorname{AKI}(p=0.19)$.

\section{Discussion}

A previous study reported secondary infection in $15 \%$ of hospitalized patients with COVID-19. ${ }^{2}$ Gram positive bacteria were the major pathogens in hospitalized (especially ventilated) patients. The rapid increase in hospitalization and ventilation, associated with COVID-19, highlighted the need for vancomycin usage in treating gram positive bacterial infections in these patients. ${ }^{1}$ Rational usage of vancomycin relies on TDM, in order to maintain an optimal concentration, and reduce the risk of treatment failure, drug resistance, as well as renal injury. Here we presented pilot findings of TDM in patients with COVID-19.

Renal dysfunction, hemodialysis and ECMO usage were major factors that affected the PK of vancomycin. ${ }^{16}$ Among eight participants with COVID-19, six (except No.1 and 5) (75\%) had at least one of these factors at baseline, implying the difficulty in the rationale of vancomycin usage among these patients. $25.4 \%$ (16/63) of serum concentration of vancomycin was beyond optimal range $(<10 \mathrm{mg} / \mathrm{L}$ at trough or $>40 \mathrm{mg} / \mathrm{L}$ at peak $)$. After treatment, $60 \%(3 / 5)$ of patients with normal baseline renal function developed acute kidney injury. These highlighted the necessity of TDM for vancomycin treatment in patients with COVID-19.

Abnormal concentration especially for peak spots was more prevalent in samples at the beginning than after initiation of TDM (vs, $P<0.05)$. After dose adjustment in three patients with abnormal trough and/or peak concentrations, it returned to and maintained within the safe and effective range. Target infection was clinically cured in 7 of the patients (one is emperical treatment), and no vancomycin-associated nephrotoxicity was detected during TDM process. TDM could be a useful tool to guide the proper usage of vancomycin in patients with COVID-19.

Although vancomycin was generally considered to be nonabsorbable through gastral administration, ${ }^{17}$ there were a few case reports of 'red man syndrome', ${ }^{18,19}$ ototoxicity or encephalopathy related to oral vancomycin. ${ }^{19,20}$ In this work, we detected a distinct and stable serum concentration for 20 days in one patient during nasal vancomycin administration alone after stopping intravenous usage. This indicated that gastral vancomycin might be absorbed. Gastral vancomycin is often used to treat or prevent Clostridium difficile infection among ventilated patients, who might be numerous in the COVID-19 pandemic. Further study and special attention is needed to determine the potential toxicity and drug resistance induced by gastral vancomycin usage in COVID-19 patients, especially those who produce detectable serum concentrations.

$\mathrm{AUC}_{0-24} / \mathrm{MIC}$ has been identified as the most suitable PK/PD index for the efficacy of vancomycin. For the MRSA infections, the recommended range of $\mathrm{AUC}_{0-24} / \mathrm{MIC}$ in the guideline is between 400 and 600 assuming a MIC of 1 $\mathrm{mg} / \mathrm{L} .{ }^{9}$ The pathogens in the COVID-19 patients in this study were MRCNS and Enterococci. Although the average 
$\mathrm{AUC}_{0-24} / \mathrm{MIC}$ of No. 1, 2 and 5 patients was less than 400, microbiological clearance was still achieved in each of them. This was consistent with the results of a prospective study in Chinese adult subjects. ${ }^{21}$ The target value of $\mathrm{AUC}_{0-24} / \mathrm{MIC}$ for clinical/ microbiological efficacy in Chinese adult patients may be between 200 and 300 . Our study showed that $\mathrm{AUC}_{0-24}$ with value $675 \mathrm{~h} \cdot \mathrm{mg} / \mathrm{L}$ may be the critical value for differentiating AKI occurrence. This was similar to a report which showed that $\mathrm{AUC}_{0-24} \geq 650 \mathrm{~h} \cdot \mathrm{mg} / \mathrm{L}$ was the cut point for AKI occurrence. ${ }^{26}$

This study had certain limitations. First, being an observational study involving only 8 patients rather than a multicenter randomized controlled trial, the data of this study should be used cautiously when applying to larger populations and different settings. Second, we found considerable serum concentration in one patient during the period of nasal vancomycin administration alone. This data along with its clinical significance need to be further verified in larger cohorts. Third, vancomycin concentration was tested with the serum samples alone, which does not best represent the concentration in key organs such as the lung and kidney. Fourth, we did not test the covariates for basic disease and concomitant usage of drugs except for antibiotics. The fitting of PPK model might be improved if these data were analyzed additionally. Fifth, Last but not least, due to the small number of participants, this study did not find a correlation between $\mathrm{AUC}_{0-24} / \mathrm{MIC}$ and efficacy in the COVID-19 patients.

\section{Conclusions}

Renal function, hemodialysis and ECMO usage were common in the COVID-19 patients, highlighting the need of TDM. An UPLC-MS/MS method was developed to quantify vancomycin concentration. Sixty-three serum samples were tested and 16 samples had a concentration beyond the expected range ( $<10$ at trough and $>40 \mathrm{mg} / \mathrm{L}$ at peak). TDM guided dosage adjustment in $37.5 \%$ of the patients, leading to an optimal concentration. All patients were cured and no vancomycin-associated nephrotoxicity was detected during the process of TDM. Considerable vancomycin concentrations were detected during sole nasal administration for 20 days, alerting the potential systemic risk during gastral usage of vancomycin. PK was consistent with two-compartment model, and CL was affected by hemodialysis and renal function. Most of the patients were infected with Staphylococcus or Enterococcus species and $\mathrm{MIC}_{90}$ was $2 \mathrm{mg} / \mathrm{L}$. Vancomycin $\mathrm{AUC}_{0-24}$ had positive correlation with AKI occurrence, while $\mathrm{AUC}_{0-24} / \mathrm{MIC}$ did not have correlation with the efficacy. It is necessary to perform randomized clinical trials to further justify the findings of the study and investigate best strategy of TDM for these patients.

\section{Methods}

\section{Study Design and Patients}

The study was performed in Shanghai Public Health Clinical Center (SPHCC, Shanghai, China), a designated hospital for COVID-19 patients. Laboratory confirmation of COVID-19 was made as previously reported. ${ }^{27}$

The clinical management of COVID-19 was adherent to the Chinese management guideline for COVID-19 (version 6.0). ${ }^{28}$ Gram-positive bacteria infection was diagnosed according to the guidelines. ${ }^{3,29}$ Cultures were carried out as described previously. ${ }^{30-32}$ Staphylococcus species were cultured in LB medium ${ }^{31}$ and Enterococcus species were in $\mathrm{BHI}$ medium. ${ }^{32}$ Vancomycin usage (initial dosage, total duration of intravenous or nasal vancomycin) was decided by an expert panel of infectious disease and critical care, in adherence to the relevant guidelines. ${ }^{3}$ Data on serum drug concentrations were sent to the clinicians within 8 hours after blood collection. Doses were adjusted by a panel of experts, based on TDM and renal function data. Pathogen clearance was defined as negative conversion of 
culture after treatment. Acute kidney injury (AKI) was defined and graded according to the KDIGO clinical practice

guidelines. ${ }^{33}$ Co-administration of nasal vancomycin to prevent Clostridium difficile colonitis was also recorded.

TDM of vancomycin was requested by the clinicians. The blood samples were collected within $0.5 \mathrm{~h}$ before the fourth continuous intravenous (IV) infusion of vancomycin (trough spot) and 0.5-1 h after infusion (peak spot). Similar time for NS administration was used. At each spot, $2 \mathrm{~mL}$ of blood was drawn into a non- anticoagulant tube, treated with acetonitrile (ACN) solution to inactivate the virus, and centrifuged. The volume of serum used for TDM was $50 \mu \mathrm{L}$ per test. The normal concentration range was set at $10-20 \mathrm{mg} / \mathrm{L}$ for the trough and $20-40 \mathrm{mg} / \mathrm{L}$ for the peak. ${ }^{10}$

The study protocol was reviewed and approved by the Ethics Commission of SPHCC (No. YJ-2020-S053-02), and all the procedures were performed in accordance with the recommendations of the Declaration of Helsinki on biomedical research involving human subjects. Informed consent was acquired from the patients or their surrogates.

\section{Measurement of Vancomycin Levels}

Vancomycin concentrations were detected by UPLC-MS/MS as previously reported. ${ }^{10}$ The details are described in the supplementary material section. Briefly, fifty microliters of serum was precipitated with $360 \mu \mathrm{L}$ acetonitrile (ACN) solution (50 $\mu \mathrm{L} 10 \%$ formic acid, $10 \mu \mathrm{L}$ demethylvacomycin (IS) $(50 \mathrm{mg} / \mathrm{L})$ and $300 \mu \mathrm{L} \mathrm{ACN})$. The operations were carried out in the BSL-2 laboratory. After precipitation, the supernatant was sent to the analytical laboratory, diluted for 20 -fold with $5 \%$ ACN solution, and detected by UPLC-MS/MS.

The UPLC system consisted of a Waters Acquity UPLC (Waters Corporation, Milford, USA) and an AB Sciex Triple Quad 5500 (AB SCIEX company, Boston, USA). Chromatographic separation of vancomycin and its IS was carried out on a Kinetex ${ }^{\circledR} 2.6 \mu \mathrm{m}$ Phenyl-Hexyl column $100 \AA$ (50 mm length x $3.0 \mathrm{~mm}$ internal diameter) (Phenomenex Company, USA). Chromatographic separation was performed using a mobile phase composed of $0.1 \% \mathrm{FA}(\mathrm{A})$ and methanol containing $0.1 \%$ FA (B). The analytes were detected by multiple reaction monitoring (MRM) mode with ion pairs of $m / z 725.5 / 144.2$ for vancomycin and $m / z 718.4 / 144.2$ for IS. The line range was 1-100 mg/L.

\section{Population PK (PPK) and pharmacokinetic / pharmacodynamic (PK/PD) analysis}

PPK model was developed to describe the time profiles of vancomycin pharmacokinetic characters using NONMEM (Ver7.4, ICON Co. Ltd, USA), PsN (Ver4.7, Uppsala University) and Xpose software (Ver4.5, Uppsala University). The base model was two-compartment model as shown in follow:

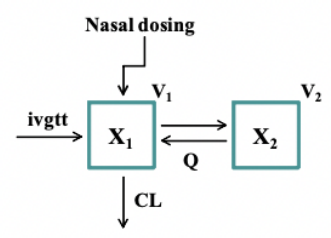

The absorption of vancomycin was consistent with zero-order kinetics. $X_{1}$ and $X_{2}$ were drug amount in the central and peripheral compartment, respectively. CL was clearance from the central compartment, while $Q$ was the intercompartment clearance between the central and peripheral compartment. $V_{1}$ and $V_{2}$ were distribution volume in the central and peripheral compartment, respectively. Model equations for evaluation of candidate covariates are as follows: 


$$
\left\{\begin{array}{l}
\frac{d X_{1}}{d t}=f_{\text {ivgtt }}+f_{\text {Nasal }}-\frac{C L}{V_{1}} X_{1}-\frac{Q}{V_{1}} X_{1}+\frac{Q}{V_{2}} X_{2} \\
\frac{d X_{2}}{d t}=\frac{Q}{V_{1}} X_{1}-\frac{Q}{V_{2}} X_{2} \\
f_{\text {ivgtt }}= \begin{cases}A M T_{\text {ivgt }} / D_{\text {ivgtt }} & t \leq D_{\text {ivgtt }} \\
0 & t>D_{\text {ivgtt }}\end{cases} \\
f_{\text {Nasal }}= \begin{cases}A M T_{\text {Nasal }} / D_{\text {Nasal }} t & t \leq D_{\text {Nasal }} \\
0 & t>D_{\text {Nasal }}\end{cases} \\
C=\frac{X_{1}}{V_{1}}
\end{array}\right.
$$

$f$ indicated input function. $\mathrm{D}_{\mathrm{Nasal}}$ and $\mathrm{D}_{\text {ivgtt }}$ indicated the duration of drug in the absorption and infusion, respectively. AMT was drug dose, and C was vancomycin concentration in central compartment. Inter-subject variability (IIV) of CL was consistent with the exponential model, while IIV of other parameters were fixed as zero. The residual error model was consistent with the proportional model.

The following covariates were tested during development of the final PPK model: gender, age, serum creatinine, estimated glomerular filtration rate, urea nitrogen, alanine aminotransferase, direct bilirubin, body temperature, hemodialysis, extracorporeal membrane oxygenation (ECMO) and concurrent use of levofloxacin and/or carpofungin. A fixed-effect model was developed using stepwise method. The covariate would be included in the model if the decrease of objective function value (OFV) was greater than $3.84(P<0.05)$ in the forward selection, or the increase of OFV was greater than $6.63(P<0.01)$ in the backward elimination. The type of covariate model tested included power model or linear model. Individual PK parameters of vancomycin were obtained using Bayesian feedback method.

The PPK model was simulated 100 times using the final estimates. Mean concentration was calculated using individual prediction data. The daily $\mathrm{AUC}_{0-24}$ was calculated using trapzoidal area method after the first dose each day. The average $\mathrm{AUC}_{0-24}$ was obtained according to sum $\left(\mathrm{AUC}_{0-24}\right)$ / (treatment duration-days without drug administration). The $\mathrm{AUC}_{0-24} / \mathrm{MIC}$ was calculated as the ratio of mean $\mathrm{AUC}_{0-24}$ to $\mathrm{MIC}$. These were performed using Matlab software (Ver7.0.1, Mathworks Co. Ltd, USA).

The correlation between $\mathrm{AUC}_{0-24} / \mathrm{MIC}$ and the microbiological effect of vancomycin was analyzed. To analyze the relationship between $\mathrm{AUC}_{0-24}$ and $\mathrm{AKI}$ occurrence, logistic regression and cross tabulation were used to find the critical value which could differentiate the AKI occurrence with maximal probability.

\section{Statistical Analysis}

Graphpad Prime 5.0 (GraphPad Software, San Diego, California) was used to compare vancomycin concentrations, and create a scatter plot. The curve of concentration for vancomycin, estimated glomerular filtration rate (eGFR) and serum creatinine level were obtained by using OriginPro 70 software (OriginLab, Massachusetts, USA). The Student $t$ test (and Nonparametric test) was used to compare the concentration levels. All tests were 2-tailed. A $P$ value $<0.05$ indicated statistical significance.

\section{Declarations}

\section{Acknowledegments}


This work was supported by the grants from National Science and Technology Program during the Thirteenth Fiveyear Plan Period (China grant, no. 2017ZX09304027, 2017ZX09304005), Shanghai Innovative treatment of novel coronavirus pneumonia (20411950200), Shanghai Research and development of emergency drugs against new coronavirus pneumonia (20431900103), and the Novel coronavirus pneumonia (COVID-19) emergency research special fund from Zhejiang University. The authors thank the experts from Renji, Ruijin, Huashan, Zhongshan, and Shanghai First and Sixth People's Hospital, as well as Shanghai Public Health Clinical Center for their clinical therapy. We thank the patients for their participation. We would like to thank proof-readers and editors for this work.

\section{Author contributions}

Lijun zhang originated, designed the study, conducted data analysis, and drafted the manuscript; Hongzhou Lu funded the research, and revised the manuscript; Lin Yin, Yuancheng Chen, Mingquan Guo, Zhaoqin Zhu, Menglu Gao, Jin Ke, Junjun Ping, and Yaru Xing contributed to sample detection and data collection; Tangkai Qi, Huichun Shi contributed to data collection, and drafted the manuscript; Yun Ling, Lin Wang, Yonghong Tao, Jing Zhang, Yaxin

Fan, Shuibao Xu, Yili Li, Xianmin Meng and Wenhong Zhang contributed to data collection. All the authors proved the final version of this manuscript.

Competing Interest All authors declared no competing interests.

\section{References}

1. Zhou F., Yu T., Du R., et al. Clinical course and risk factors for mortality of adult inpatients with COVID-19 in Wuhan, China: a retrospective cohort study. Lancet. 2020; 395(10229):1054-1062.

2. Erb C.T., Patel B., Orr J.E., et al. Management of Adults with Hospital-acquired and Ventilator-associated Pneumonia. Ann Am Thorac Soc. 2016; 13(12):2258-2260.

3. Kalil A.C., Metersky M.L., Klompas M., et al. Management of Adults With Hospital-acquired and Ventilatorassociated Pneumonia: 2016 Clinical Practice Guidelines by the Infectious Diseases Society of America and the American Thoracic Society. Clin Infect Dis. 2016; 63(5):e61-e111.

4. Watkins R.R.凶Deresinski S. Increasing Evidence of the Nephrotoxicity of Piperacillin/Tazobactam and Vancomycin Combination Therapy-What Is the Clinician to Do? Clin Infect Dis. 2017; 65(12):2137-2143.

5. Liang X., Fan Y., Yang M., et al. A Prospective Multicenter Clinical Observational Study on Vancomycin Efficiency and Safety With Therapeutic Drug Monitoring. Clin Infect Dis. 2018; 67(suppl_2):S249-S255.

6. Hori Y., Aoki N., Kuwahara S., et al. Megalin Blockade with Cilastatin Suppresses Drug-Induced Nephrotoxicity. $J$ Am Soc Nephrol. 2017; 28(6):1783-1791.

7. Rybak M.J., Rotschafer J.C.खRodvold K.A. Vancomycin: over 50 years later and still a work in progress. Pharmacotherapy. 2013; 33(12):1253-1255.

8. Doernberg S.B., Lodise T.P., Thaden J.T., et al. Gram-Positive Bacterial Infections: Research Priorities, Accomplishments, and Future Directions of the Antibacterial Resistance Leadership Group. Clin Infect Dis. 2017; 64(suppl_1):S24-S29.

9. Rybak M.J., Le J., Lodise T.P., et al. Therapeutic monitoring of vancomycin for serious methicillin-resistant Staphylococcus aureus infections: A revised consensus guideline and review by the American Society of HealthSystem Pharmacists, the Infectious Diseases Society of America, the Pediatric Infectious Diseases Society, and the Society of Infectious Diseases Pharmacists. Am J Health Syst Pharm. 2020. 
10. Fan Y., Peng X., Yu J., et al. An ultra-performance liquid chromatography-tandem mass spectrometry method to quantify vancomycin in human serum by minimizing the degradation product and matrix interference.

Bioanalysis. 2019; 11(10):941-955.

11. Rybak M., Lomaestro B., Rotschafer J.C., et al. Therapeutic monitoring of vancomycin in adult patients: a consensus review of the American Society of Health-System Pharmacists, the Infectious Diseases Society of America, and the Society of Infectious Diseases Pharmacists. Am J Health Syst Pharm. 2009; 66(1):82-98.

12. Matsumoto K., Takesue Y., Ohmagari N., et al. Practice guidelines for therapeutic drug monitoring of vancomycin: a consensus review of the Japanese Society of Chemotherapy and the Japanese Society of Therapeutic Drug Monitoring. J Infect Chemother. 2013; 19(3):365-380.

13. Steinmetz T., Eliakim-Raz N., Goldberg E., Leibovici L. $₫$ Yahav D. Association of vancomycin serum concentrations with efficacy in patients with MRSA infections: a systematic review and meta-analysis. Clin Microbiol Infect. 2015; 21(7):665-673.

14. Ye Z.K., Chen Y.L., Chen K., et al. Therapeutic drug monitoring of vancomycin: a guideline of the Division of Therapeutic Drug Monitoring, Chinese Pharmacological Society. J Antimicrob Chemother. 2016; 71(11):30203025.

15. Delgado A., Mejia I., Barreto E., et al. Experience in Therapeutic Monitoring of Vancomycin in Adult Patients Undergoing Continuous Ambulatory Peritoneal Dialysis (Capd), 2010-2012. Basic Clin Pharmacol. 2012; 111:3131.

16. Vickers R.J., Tillotson G.S., Nathan R., et al. Efficacy and safety of ridinilazole compared with vancomycin for the treatment of Clostridium difficile infection: a phase 2, randomised, double-blind, active-controlled, non-inferiority study. The Lancet Infectious diseases. 2017; 17(7):735-744.

17. Bryan C.S.खWhite W.L. Safety of oral vancomycin in functionally anephric patients. Antimicrob Agents Chemother. 1978; 14(4):634-635.

18. Bergeron L.『Boucher F.D. Possible red-man syndrome associated with systemic absorption of oral vancomycin in a child with normal renal function. Ann Pharmacother. 1994; 28(5):581-584.

19. Cimolai N. Does oral vancomycin use necessitate therapeutic drug monitoring? Infection. 2020; 48(2):173-182.

20. Gomceli U., Vangala S., Zeana C., Kelly P.J.囚Singh M. An Unusual Case of Ototoxicity with Use of Oral Vancomycin. Case Rep Infect Dis. 2018; 2018:2980913.

21. Westra N., Proost J.H., Franssen C.F.M., et al. Vancomycin pharmacokinetic model development in patients on intermittent online hemodiafiltration. PLoS One. 2019; 14(5):e0216801.

22. Shen K., Yang M., Fan Y., et al. Model-based Evaluation of the Clinical and Microbiological Efficacy of Vancomycin: A Prospective Study of Chinese Adult In-house Patients. Clin Infect Dis. 2018; 67(suppl_2):S256S262.

23. Li D., Lv P., Fan L., et al. The immobilization of antibiotic-loaded polymeric coatings on osteoarticular Ti implants for the prevention of bone infections. Biomater Sci. 2017; 5(11):2337-2346.

24. Donadello K., Roberts J.A., Cristallini S., et al. Vancomycin population pharmacokinetics during extracorporeal membrane oxygenation therapy: a matched cohort study. Crit Care. 2014; 18(6):632.

25. Hahn J., Choi J.H.खChang M.J. Pharmacokinetic changes of antibiotic, antiviral, antituberculosis and antifungal agents during extracorporeal membrane oxygenation in critically ill adult patients. J Clin Pharm Ther. 2017; 42(6):661-671.

26. Aljefri D.M., Avedissian S.N., Rhodes N.J., et al. Vancomycin Area Under the Curve and Acute Kidney Injury: A Meta-analysis. Clin Infect Dis. 2019; 69(11):1881-1887. 
27. Huang C., Wang Y., Li X., et al. Clinical features of patients infected with 2019 novel coronavirus in Wuhan, China. Lancet. 2020; 395(10223):497-506.

28. Lodise T.P., Rosenkranz S.L., Finnemeyer M., et al. The Emperor's New Clothes: Prospective Observational Evaluation of the Association between Initial Vancomycin Exposure and Failure Rates among Adult Hospitalized Patients with MRSA Bloodstream Infections (PROVIDE). Clin Infect Dis. 2019.

29. Baron E.J., Miller J.M., Weinstein M.P., et al. A guide to utilization of the microbiology laboratory for diagnosis of infectious diseases: 2013 recommendations by the Infectious Diseases Society of America (IDSA) and the American Society for Microbiology (ASM)(a). Clin Infect Dis. 2013; 57(4):e22-e121.

30. Qi T., Zhang R., Shen Y., et al. Etiology and clinical features of 229 cases of bloodstream infection among Chinese HIV/AIDS patients: a retrospective cross-sectional study. Eur J Clin Microbiol Infect Dis. 2016; 35(11):1767-1770.

31. Guo M., Feng C., Ren J., et al. A Novel Antimicrobial Endolysin, LysPA26, against Pseudomonas aeruginosa. Front Microbiol. 2017; 8:293.

32. Cheng M., Zhang Y., Li X., et al. Endolysin LysEF-P10 shows potential as an alternative treatment strategy for multidrug-resistant Enterococcus faecalis infections. Sci Rep. 2017; 7(1):10164.

33. Khwaja A. KDIGO clinical practice guidelines for acute kidney injury. Nephron Clin Pract. 2012; 120(4):c179-184.

\section{Tables}

\section{Table 1 The clinical and experimental characters of 8 patients}

\begin{tabular}{|c|c|c|c|c|c|c|c|c|c|c|c|c|c|c|c|c|c|c|c|c|}
\hline No. & $\begin{array}{l}\text { Age } \\
\text { (Year) }\end{array}$ & Sex & Pathogen & $\begin{array}{c}\mathrm{MIC} \\
\mathrm{mg} / \mathrm{L}\end{array}$ & $\begin{array}{l}\text { Infection } \\
\text { site }\end{array}$ & ECMO H & $\begin{array}{c}\text { Hemodyalysis } \\
\text { on Baseline }\end{array}$ & $\begin{array}{l}\text { s Baseline } \\
\text { Creatinine }\end{array}$ & $\begin{array}{c}\text { Baseline } \\
\text { eGFR }\end{array}$ & $\begin{array}{l}\text { e Initial } \\
\text { Dose }\end{array}$ & $\begin{array}{c}\text { Nasal feed } \\
\text { vancomycin }\end{array}$ & $\begin{array}{c}\text { Therapy } \\
\text { duration } \\
\text { (Total/IV/Pre.)* }\end{array}$ & $\begin{array}{l}\text { Sub- } \\
\text { optimal/ } \\
* \quad \text { toatl } \\
\text { troughs }\end{array}$ & $\begin{array}{c}\text { Overdose/ } \\
\text { total } \\
\text { peaks }\end{array}$ & / Ctrough/Cpeak & $\begin{array}{l}\text { Adjust } \\
\text { dose }\end{array}$ & $\begin{array}{l}\text { st Pathogen } \\
\text { Clearance }\end{array}$ & & $\begin{array}{l}\text { I AUC0- } \\
24 \\
(\mathrm{~h} \cdot \mathrm{mg} / \mathrm{L})\end{array}$ & $\begin{array}{l}\text { AUC0- } \\
\text { 24/MIC } \\
\text { (h) }\end{array}$ \\
\hline 1 & 64 & $\mathrm{M}$ & $\begin{array}{l}\text { Staphylococcus } \\
\text { haemolyticus }\end{array}$ & 2 & Lung & Yes & No & 71.77 & 101.39 & $\begin{array}{l}\text { 1000mg } \\
\text { Q12h }\end{array}$ & $250 \mathrm{mg}$ Q6h & $28 / 28 / 5$ & $4 / 16$ & $2 / 14$ & $\begin{array}{c}4.63-23.6 / 17- \\
47.7\end{array}$ & Yes & Yes & 1 & 676 & 338 \\
\hline 2 & 81 & M & $\begin{array}{l}\text { Enterococcus } \\
\text { faecalis }\end{array}$ & 2 & $\begin{array}{l}\text { Blood- } \\
\text { stream }\end{array}$ & Yes & Yes & 188.67 & 31.7 & $\begin{array}{l}\text { 1000mg } \\
\text { Q12h }\end{array}$ & $250 \mathrm{mg} \mathrm{Q6h}$ & $39 / 5 / 3$ & $5 / 13$ & $3 / 9$ & $\begin{array}{c}5.9-34.2 / 21.8- \\
49.9\end{array}$ & Yes & Yes & NA & 488 & 244 \\
\hline 3 & 62 & M & $\begin{array}{l}\text { Enterococcus } \\
\text { faecium }\end{array}$ & $\leq 0.5$ & Lung & Yes & No & 77.4 & 92 & $\begin{array}{l}\text { 1000mg } \\
\text { Q12h }\end{array}$ & No & $25 / 25 / 4$ & $0 / 1$ & $0 / 0$ & $6.15-18.8 / \mathrm{NA}$ & No & Yes & NA & 456 & 912 \\
\hline 4 & 75 & M & $\begin{array}{l}\text { Enterococcus } \\
\text { faecium }\end{array}$ & $\leq 0.5$ & Lung & Yes & Yes & 85.62 & 80.13 & $\begin{array}{l}500 \mathrm{mg} \\
\text { Q8h }\end{array}$ & 250mg Q6h & $17 / 13 / 4$ & $0 / 3$ & $1 / 1$ & $19.5-26.6 / 41.7$ & Yes & Yes & 3 & 869 & 1738 \\
\hline 5 & 57 & F & $\begin{array}{l}\text { Staphylococcus } \\
\text { haemolyticus }\end{array}$ & 1 & $\begin{array}{l}\text { Blood- } \\
\text { stream }\end{array}$ & No & No & 46.88 & 107.93 & $\begin{array}{l}\text { 1000mg } \\
\text { Q12h }\end{array}$ & $250 \mathrm{mg} \mathrm{Q6h}$ & $13 / 12 / 2$ & $0 / 1$ & $1 / 1$ & $12.1 / 41.43$ & No & Yes & NA & 370 & 370 \\
\hline 6 & 70 & M & $\begin{array}{l}\text { Staphylococcus } \\
\text { haemolyticus }\end{array}$ & 1 & Lung & No & Yes & 79.82 & 88.09 & $\begin{array}{l}\text { 1000mg } \\
\text { Q12h }\end{array}$ & No & $11 / 11 / 3$ & $0 / 1$ & $0 / 0$ & $15.1 / \mathrm{NA}$ & No & Yes & NA & 984 & 984 \\
\hline 7 & 63 & $\mathrm{~F}$ & $\begin{array}{l}\text { Emperical } \\
\text { (NA) }\end{array}$ & NA & Lung & Yes & No & 31.67 & 193.99 & $\begin{array}{l}\text { 1000mg } \\
\text { Q12h }\end{array}$ & $\begin{array}{l}\text { 125mg } \\
\text { Q6h }\end{array}$ & $11 / 10 / 6$ & $0 / 1$ & $0 / 1$ & $14.3 / 34.8$ & No & NA & 2 & 457 & NA \\
\hline 8 & 65 & M & $\begin{array}{l}\text { Enterococcus } \\
\text { faecium }\end{array}$ & $\leq 0.5$ & Lung & No & Yes & 146.48 & 43.37 & $\begin{array}{l}\text { 1000mg } \\
\text { Q12h }\end{array}$ & No & $5 / 5 / 5$ & $0 / 0$ & $0 / 1$ & $\mathrm{NA} / 24.6$ & No & Yes & NA & 674 & 1349 \\
\hline Mean & & & & & & & & & & & & & & & & & & & 622 & 848 \\
\hline SD & & & & & & & & & & & & & & & & & & & 218 & 566 \\
\hline
\end{tabular}

* Therapy duration (Total/IV/Pre.) represents the days for total vancomycin therapy / IV administration / the days before drug concentration detection, respectively

AKI: acute kidney injury

ECMO: Extracorporeal Membrane Oxygenation 
NA: not available. For AKI data, it means AKI did not occur

SD: standard deviation

Table 2 Vancomycin PK parameters in the final PPK model

\begin{tabular}{llc}
\hline Parameter (Unit) & Explain & Value (RSE\%) \\
\hline $\mathrm{CL}(\mathrm{L} / \mathrm{h})$ & Clearance from central compartment & $4.33(23.8 \%)$ \\
$\mathrm{V}_{1}(\mathrm{~L})$ & Distribution volume in central compartment & $2.00(62.3 \%)$ \\
$\mathrm{Q}(\mathrm{L} / \mathrm{h})$ & Inter-compartment clearance between central and peripheral compartment & $4.14(68.4 \%)$ \\
$\mathrm{V}_{2}(\mathrm{~L})$ & Distribution volume in peripheral compartment & $56.7(36.3 \%)$ \\
$\mathrm{D}_{1}(\mathrm{~h})$ & Duration during drug absorption & $1.90(6.0 \%)$ \\
$\theta_{\mathrm{Hemo}}$ & Impact factor of hemodialysis on the clearance & $0.42(13.5 \%)$ \\
$\theta_{\text {Scr }}$ & Impact factor of serum creatinine on the clearance & $0.41(20.5 \%)$ \\
$\varepsilon(\%)$ & Proportional residual error item & $31.3(5.7 \%)$ \\
\hline
\end{tabular}

RSE: relative standard error

\section{Figures}



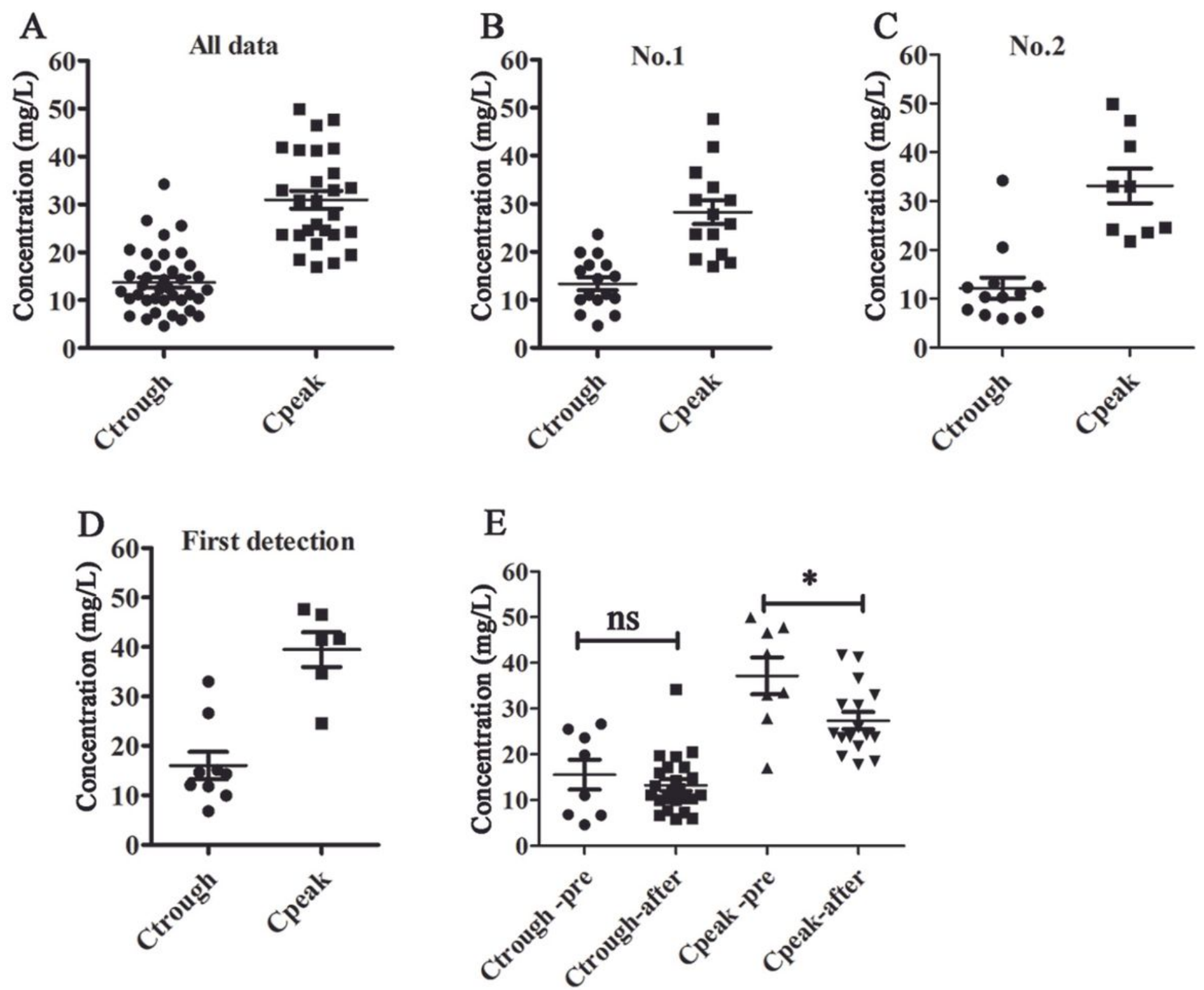

Figure 1

Monitoring of serum vancomycin concentration in COVID-19 patients A. All data from 8 patients. B and C. The data from patient No.1 and patient No.2, respectively. D. The data from the first detection of all patients. E. Comparison between the concentration before and after dose adjustments. $\mathrm{P}<0.05$ represents statistical difference. ns, no statistical difference. *, $\mathrm{p}<0.05$. 
A
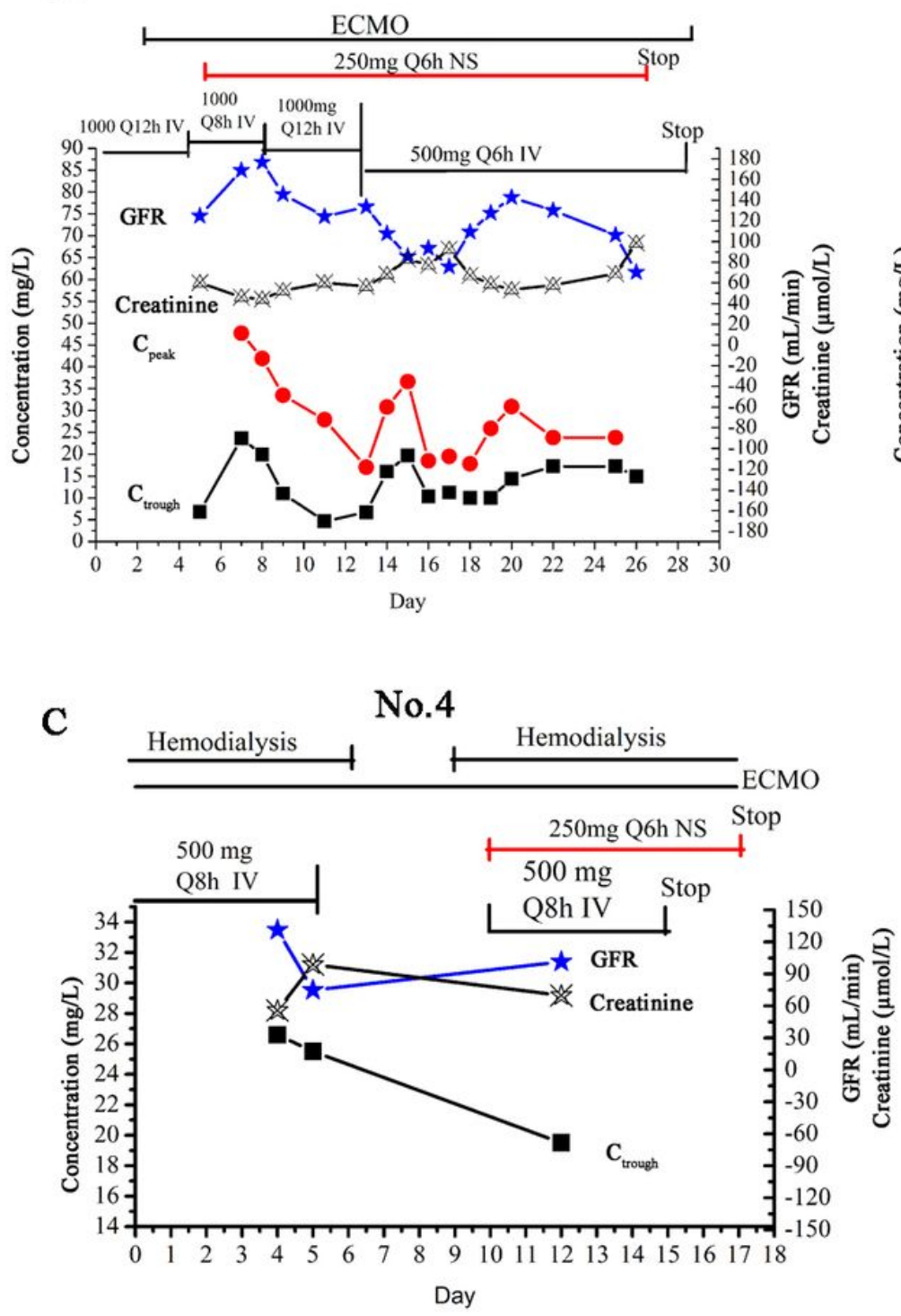

B

No.2

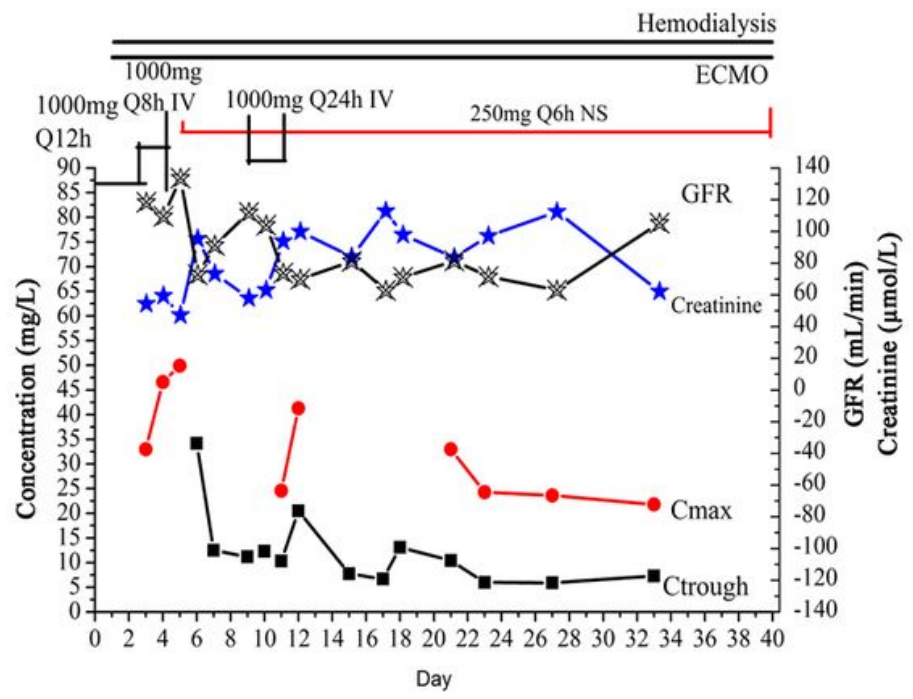

Figure 2

The curve of concentration for vancomycin, GFR, and creatinine from three patients with dose adjustments A. from No. 1; B. from No.2; C. from No.4. Intravenous administration (IV) shown in dosing and blank line; Nasal feed (NS) shown in dosing and red line. Square frame and blank curve: Ctrough; Circular dots and red line: Cpeak; Star and blue line: GFR; Star and blank line: Creatinine. The date giving loading dose was counted as day 0, and the data for vancomycin stopping as"stop". ECMO and Hemodialysis were shown from loading dose to drug stopping. 

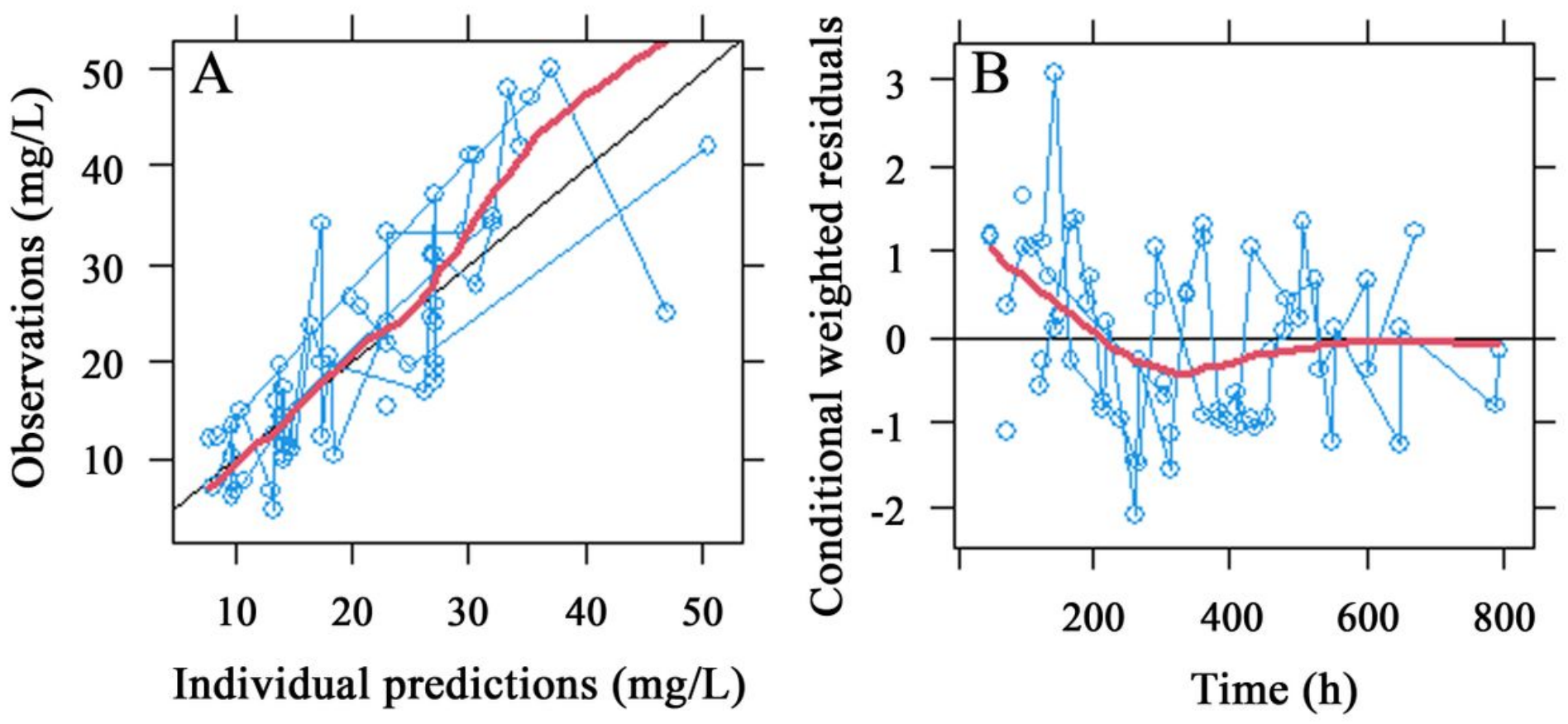

Figure 3

Diagnostic plot of final PPK model of vancomycin Circles means actual data. Red line means local weighted regression line, while blank line means unity line $(A)$ or zero horizontal line (B).

\section{Supplementary Files}

This is a list of supplementary files associated with this preprint. Click to download.

- Supplementmethodsandresultsvancomycinrevised0616.docx 